\title{
Osteochondral repair with synovial membrane-derived mesenchymal stem cells
}

\author{
HONG LI $^{1}$, JUNCHAO QIAN ${ }^{2}$, JIWU CHEN $^{1}$, KAI ZHONG ${ }^{2}$ and SHIYI CHEN ${ }^{1}$ \\ ${ }^{1}$ Department of Sports Medicine, Huashan Hospital, Shanghai 200040; ${ }^{2}$ High Magnetic Field Laboratory, \\ Chinese Academy of Sciences, Hefei, Anhui 230031, P.R. China
}

Received January 19, 2015; Accepted November 10, 2015

DOI: $10.3892 / \mathrm{mmr} .2016 .4795$

\begin{abstract}
The aim of the present study was to analyze cartilage repair tissue quality following synovial membrane-derived mesenchymal stem cell (SMSC) transplantation in a rabbit osteochondral defect. A total of 15 New Zealand white rabbits were randomly distributed into three groups ( $\mathrm{n}=5$ in each group). In group 1, an osteochondral defect model was established in the right knee trochlea, prior to transplantation with SMSCs (SMSC group). In group 2, an osteochondral defect model was established without further treatment (control group). Group 3 did not undergo osteochondral defect model establishment and served as the sham control (normal group). All animals were sacrificed 12 weeks following the surgical procedures for magnetic resonance imaging and histological examination. No significant differences were observed between the control and SMSC group in the macroscopic score $(\mathrm{P}>0.05)$, the $2 \mathrm{D}$ magnetic resonance observation of cartilage repair tissue score $(\mathrm{P}>0.05)$ or the modified O'Driscoll scale $(\mathrm{P}>0.05)$. Compared with the control group, a significant improvement in tissue quality was observed in the SMSCs group postoperatively. The repair tissue of the SMSCs group had a shorter $\mathrm{T}_{2}$, compared with that of the control group, although no significant difference was detected $(\mathrm{P}>0.05)$. Furthermore, the apparent diffusion coefficient in the repair tissue of the SMSC group had a significantly lower value, compared with that of the control group $(\mathrm{P}=0.016)$. The results of the present study demonstrated that osteochondral repair using SMSCs facilitated the repair of appropriate tissue texture.
\end{abstract}

Correspondence to: Professor Shiyi Chen, Department of Sports Medicine, Huashan Hospital, 12 Wulumuqi Zhong Road, Shanghai 200040, P.R. China

E-mail: cshiyi@163.com

Professor Kai Zhong, High Magnetic Field Laboratory, Chinese Academy of Sciences, 350 Shushanhu Road, Hefei, Anhui 230031, P.R. China

E-mail: kzhong@hmfl.ac.cn

Key words: cartilage, synovial mesenchymal stem cells, magnetic resonance imaging

\section{Introduction}

Cartilage lesions in the joints remain a major clinical challenge due to their poor healing ability following injury. At present, the most commonly used treatments comprise microfracture, osteochondral autologous transplantation and autologous chondrocyte implantation, which can provide short-term success $(1,2)$. Previously, mesenchymal stem cells (MSCs) have been suggested as an alternative cell-source for cartilage regeneration, due to their ease of isolation and amenability to ex vivo expansion (3-5). Therefore, MSCs may provide a cell source for novel treatment strategies in cartilage regeneration (4). Histologically, it has been observed that autologous matrix-assisted MSC implantation generates significantly more cartilage matrix into cartilage defects in animal experiments $(6,7)$. However, histological evaluation of cartilage repair tissue is invasive, and a non-invasive method is required to systematically evaluate the integrity of the repair tissue.

Magnetic resonance imaging (MRI), as a non-invasive approach, has been widely adapted to examine cartilage and repair tissue (8-10). In addition, advanced high-field MRI techniques can generate images with high spatial resolution, which enables visualization of the regenerated repair tissue in more detail $(11,12)$. Qualitative $T_{2}$ mapping is able to differentiate hyaline cartilage from repair tissues, and assess collagen fibril network organization of the native hyaline cartilage following cartilage repair procedures (13-15). Furthermore, diffusion-weighted imaging (DWI) is able to detect changes in water mobility within the cartilage repair tissue, and may provide a method of quantification of cartilage maturation and tissue quality $(16,17)$. Therefore, additional information regarding cartilage repair tissue can be obtained with $\mathrm{T}_{2}$-mapping and DWI, in addition to standard morphological MRI (18).

In the present study, the quality of cartilage repair tissue following SMSC treatment was investigated in a rabbit large osteochondral defect model using routine morphological MRI, $\mathrm{T}_{2}$ mapping and a DWI MRI technique with a 9.4T high-field. It was hypothesized that osteochondral repair using SMSCs facilitates the repair of appropriate tissue structure.

\section{Materials and methods}

Harvesting synovial cells from rabbits. In the present study, three New Zealand white rabbits (male, 5 months old, 
weighing $\sim 3 \mathrm{~kg}$ ) were used as the source of synovial cells. Rabbits were maintained at $20-25^{\circ} \mathrm{C}$ in a $12 / 12 \mathrm{~h}$ light/dark cycle. Synovium-derived MSCs (SMSCs) were isolated and expanded, as previously described (19). The rabbits were anesthetized with pentobarbital (Sigma-Aldrich, St. Louis, MO, USA) and the synovial tissue $(1 \times 1 \times 1 \mathrm{~cm})$ was harvested from the knee joint. Harvested synovial tissue samples were finely sectioned with scissors and digested with $0.02 \%$ collagenase (Sigma-Aldrich) in high-glucose Dulbecco's modified Eagle's medium (DMEM, GE Healthcare Life Sciences, Chalfont, UK) supplemented with $10 \%$ fetal bovine serum (FBS, Gibco; Thermo Fisher Scientific, Inc., Waltham, MA, USA) and antibiotics $(100 \mathrm{U} / \mathrm{ml}$ penicillin and $100 \mathrm{U} / \mathrm{ml}$ streptomycin; GE Healthcare Life Sciences). Following overnight incubation at $37^{\circ} \mathrm{C}$, the cells were collected by centrifugation $(1,000 \mathrm{x} \mathrm{g}$ for $5 \mathrm{~min}$ at room temperature), washed twice with phosphate-buffered saline (PBS), and resuspended in high-glucose DMEM supplemented with $10 \%$ FBS and antibiotics. The SMSCs were then seeded into culture flasks $\left(1 \times 10^{5}\right.$ cells $\left./ \mathrm{ml}\right)$ and incubated in complete medium (low-glucose DMEM supplemented with $10 \% \mathrm{FBS}$ and antibiotics) at $37^{\circ} \mathrm{C}$ in a humidified atmosphere containing $5 \% \mathrm{CO}_{2}$ for proliferation. The complete medium was replaced once every 3 days. When the attached cells reached $90 \%$ confluence, following 9-12 days of primary culture, the cells were washed twice with sterilized PBS solution, collected by treatment with trypsin-EDTA $(0.25 \%$ trypsin and $1 \mathrm{mM}$ EDTA; Cell Applications, Inc., San Diego, CA USA), and seeded in new culture flasks at a dilution of 1:4 for the first sub-culture. The medium was replaced after 2 days to allow cell adhesion and to remove the adherent cells.

Animal experiments. All animal experiments were approved by the internal Animal Care and Use Committee at Shanghai Jiaotong University (Shanghai, China). A total of 15 white New Zealand rabbits (male, 5 months old, weighing $2.7 \pm 0.5 \mathrm{~kg}$ ) were used for the present study. In the normal group $(n=5)$, a surgical incision was introduced in the right knee joint without osteochondral defect model establishment. The remaining 10 rabbits underwent a surgical procedure to establish an osteochondral defect in the right knee. Among these, five rabbits subsequently received SMSC therapy (SMSC group), whereas the remaining five rabbits received no further treatment (control group). All animals underwent anesthesia by intravenous administration of $3 \%$ pentobarbital $(30 \mathrm{mg} / \mathrm{kg})$. Briefly, the animal was placed in a supine position on the operative table. Following skin preparation and disinfection, a midline incision was made, and a lateral parapatellar arthrotomy was performed to expose the femoral trochlea. A round full-thickness cartilage defect, $6 \mathrm{~mm}$ in diameter and $3 \mathrm{~mm}$ in depth, was created in the central portion of the femoral trochlea groove using a ring-drill (Osteochondral Autograft Transfer System; Arthrex, Inc., Naples, FL, USA). In the SMSC group, the defect was injected with the $1 \%$ high availability solution (Shanghai YuanYe Biological Technology Co., Ltd., Shanghai, China) containing SMSCs $\left(2 \times 10^{5}\right.$ cells $/ \mathrm{ml}$, $0.5 \mathrm{ml} /$ defect). Following treatment, the patella was reduced, the joint capsule was closed with interrupted sutures and the wound was closed in layers. Postoperatively, the animals were returned to their cages and allowed free cage activity without immobilization.

All rabbits were sacrificed 12 weeks following the surgical procedures for examination, using an overdose of pentobarbital. The macroscopic appearance of the defects was scored using a newly developed semi-quantitative macroscopic scoring system, developed by Goebel et al (8). This reverse scale consists of five major parameters, and a total of 20 points indicates the worst possible result.

Evaluation by 9.4 T high-field MRI. Immediately following sacrifice, knee tissue samples were harvested for MRI examination. MRI scans were performed on a 9.4T/400 mm wide bore MRI scanner (Agilent DD2 NMR console; Agilent Technologies, Inc., Santa Clara, CA, USA) using a volume $\mathrm{RF}$ coil (inner diameter, $40 \mathrm{~mm}$; Bruker Corporation, Billerica, MA, USA). A 3D spoiled gradient echo (GRE) sequence was selected to perform isovolumetric scans of the osteochondral samples. Minimum voxel size was set to $125 \times 86 \times 86 \mu \mathrm{m}$, and optimized imaging parameters were evaluated as: Repetition time (TR), $5.4 \mathrm{~ms}$; time echo (TE), $3 \mathrm{~ms}$; flip angle (FA), $7^{\circ}$; number of excitations (NEX), 9 and bandwidth (BW), $50 \mathrm{kHz}$. The cartilage defects were evaluated using a $2 \mathrm{D}$ magnetic resonance observation of cartilage repair tissue (MOCART) score (20). Qualitative $\mathrm{T}_{2}$ maps were collected using a multi-echo multi-slice sequence $(\mathrm{TR}=2,500 \mathrm{~ms}, \mathrm{TE}=10 \mathrm{~ms}, \mathrm{NEX}=1$, $\mathrm{FOV}=25 \mathrm{~mm}{ }^{*} 25 \mathrm{~mm}$, matrix $=256 \times 256$, slice thickness $=1 \mathrm{~mm}$ ). Diffusion-weighted imaging was selected using a spin echo sequence $(\mathrm{TR} / \mathrm{TE}=2,300 / 36.5 \mathrm{~ms}$, $\mathrm{NEX}=1$, matrix $=128 \times 128, \mathrm{FOV}=25 \mathrm{~mm}{ }^{*} 25 \mathrm{~mm}$, section thickness $=1 \mathrm{~mm}$ ). Pixel-wise calculation of the apparent diffusion coefficient maps was performed numerically.

Histology evaluation. Following MRI scans, all samples were fixed in $10 \%$ neutral-buffered formalin solution (Wuhan Goodbio Technology Co., Ltd., Wuhan, China) for 2 days, and the samples were then decalcified in 10\% EDTA solution for $\sim 4$ weeks at room temperature. The samples were sectioned $(5 \mu \mathrm{m})$ through the center of the defect using a microtome (SM2500; Leica Microsystems GmbH, Mannheim, Germany), and these sections were stained with hematoxylin and eosin (Wuhan Goodbio Technology Co., Ltd.) and Safranin-O (Sigma-Aldrich). The slides were visualized using inverted light microscopy (IX71SBF-2; Olympus Corporation, Tokyo, Japan). Digital images were captured using a DP Manager camera(Olympus Corporation). Two investigators performed histological analysis in a blinded-manner, in which five histological sections in each group were scored according to the modified O'Driscoll scale (21), with a lower score indicating lower histological quality of the cartilage repair tissue.

Data analysis. Statistical analysis was performed using Stata 10.0 software (Stata Corp, College Station, TX, USA), and data are presented as the mean \pm standard deviation. Statistical analysis of the quantitative results was performed using one-way analysis of variance or a Kruskal-Wallis test. $\mathrm{P}<0.05$ was considered to indicate a statistically significant difference. 


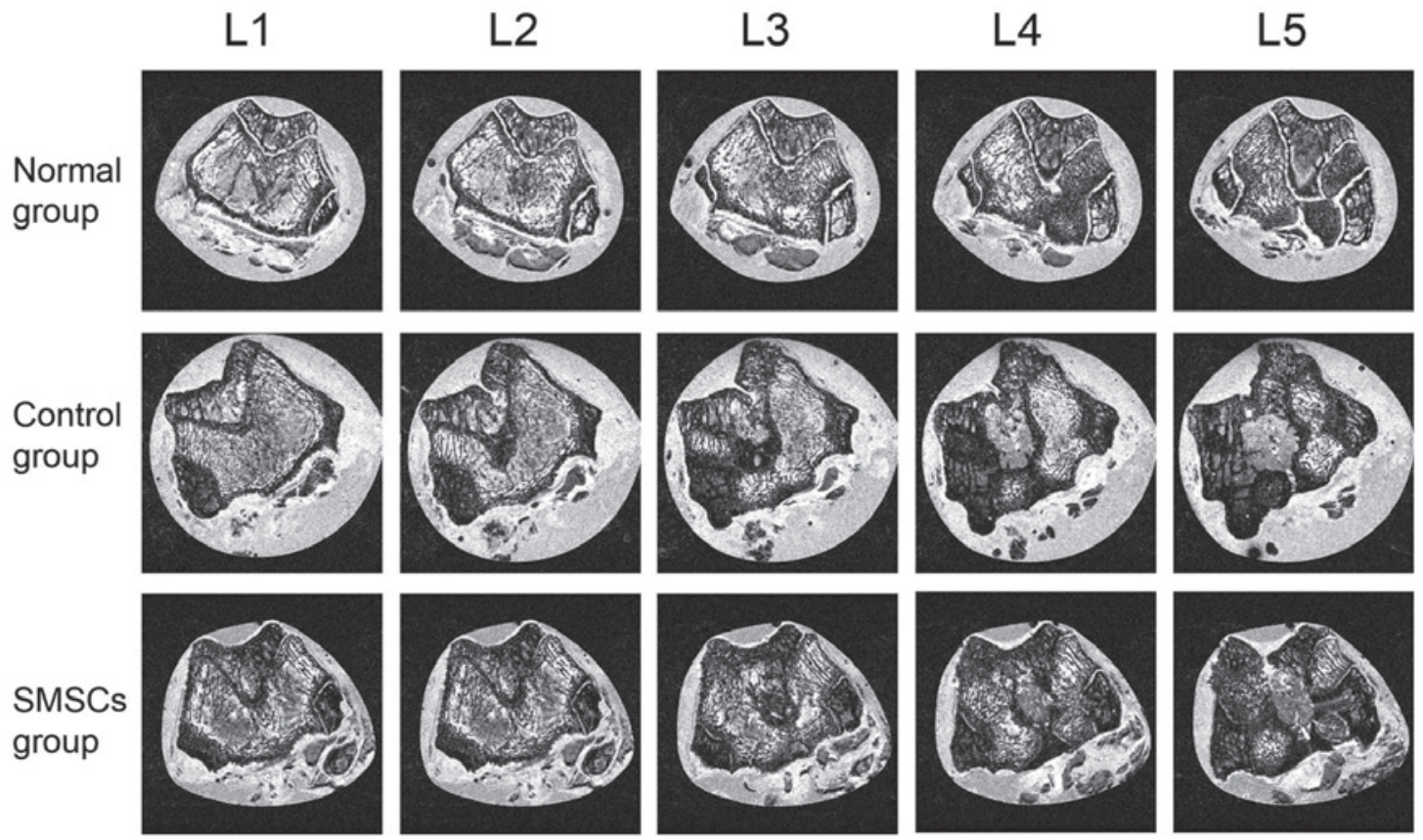

Figure 1. High-spatial-resolution morphologic magnetic resonance images of continuous cross sections of articular cartilage. L1-5 represents consecutive scan slice levels, revealing the cartilage defect. SMSCs, synovial membrane-derived mesenchymal stem cells.

\section{Results}

Macroscopic results. No complications were observed in the rabbits throughout the course of the present study. At 12 weeks following surgery, the macroscopic appearance of the control group showed complete defect filling. The surface of the repair tissue was not flat, compared with that of the normal group. In four samples of the SMSC group, virtually complete defect filling was observed, whilst one sample of the SMSCs group exhibited incomplete defect filling with a severe fissure. For all samples of the SMSC group, the appearance of the repair tissue was similar to that of the adjacent cartilage tissue.

MRI results. The GRE images captured of the continuous cross section of articular cartilage and cartilage-bone interface are shown in Fig. 1. In the normal group, the surface appearance and signal intensity throughout the cartilage were flat, and the tide mark was clearly visible. In the control group, defect filling was complete and the defect remained visible. The signal intensity of the repair tissue was also abnormal, and the subchondral lamina was not intact. In addition, the signal intensity of the osteochondral defect site was higher, compared with that of the adjacent cartilage. In the single sample with severe fissure in the SMSC group, the osteochondral site revealed a similar pattern as the control group sample, with high signal intensity at the site of repair (data not shown). For the remaining samples of the SMSC group, the defect filling was almost complete, and a demarcation border was visible. The signal intensity of the repair tissue was almost normal, and the subchondral lamina was intact. Granulation tissue was also observed in the subchondral bone in the SMSC group, and the signal intensity of the granulation tissue was continuous, compared with that of the adjacent cartilage.

The results of the $\mathrm{T}_{2}$ mapping for each group are shown in Fig. 2. In the normal group, $\mathrm{T}_{2}$ mapping of the cartilage revealed

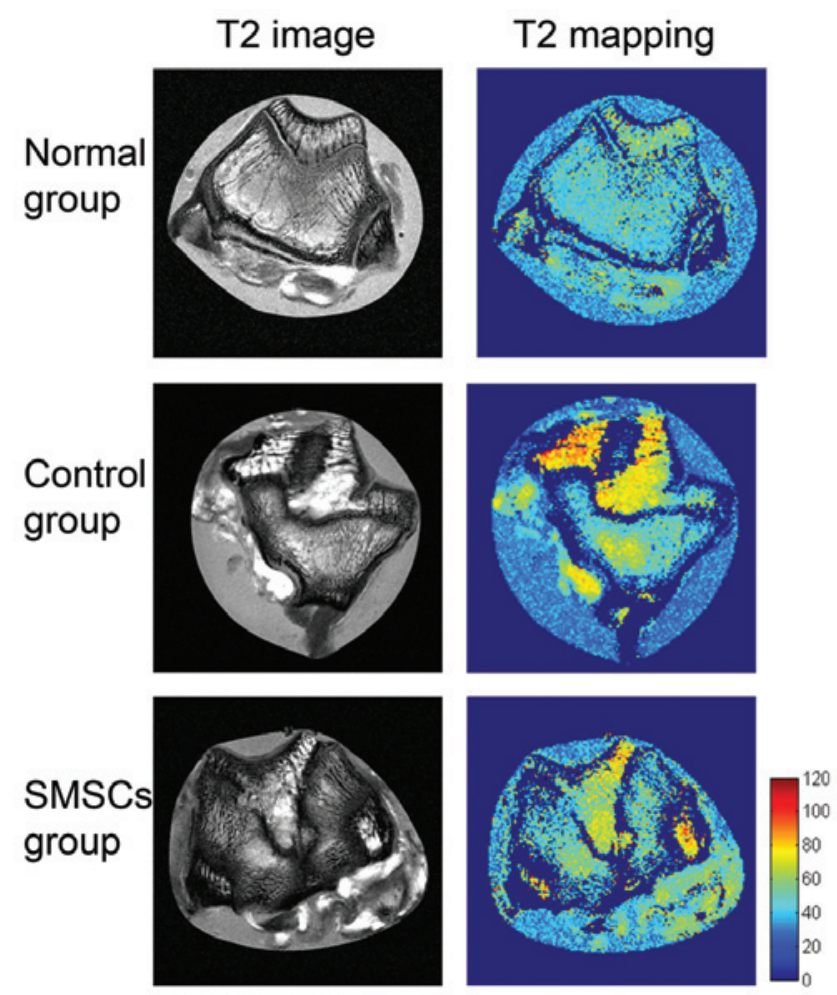

Figure 2. $\mathrm{T}_{2}$ map of cartilage repair tissue in the normal group, OCD group and SMSC group. Higher color values indicate areas of poor cartilage repair. OCD, osteochondral defect; SMSC, synovial membrane-derived mesenchymal stem cells.

a homogenous $T_{2}$ pattern, with no extensive signal variation between the deep and the superficial cartilage. In the control group, the $T_{2}$ values in the repair tissue were significantly higher, compared with those in the normal group $(52.48 \pm 1.60$, vs. $28.60 \pm 3.20 \mathrm{~ms} ; \mathrm{P}=0.009$; Fig. $3 \mathrm{~A}$ ). The $\mathrm{T}_{2}$ mapping 


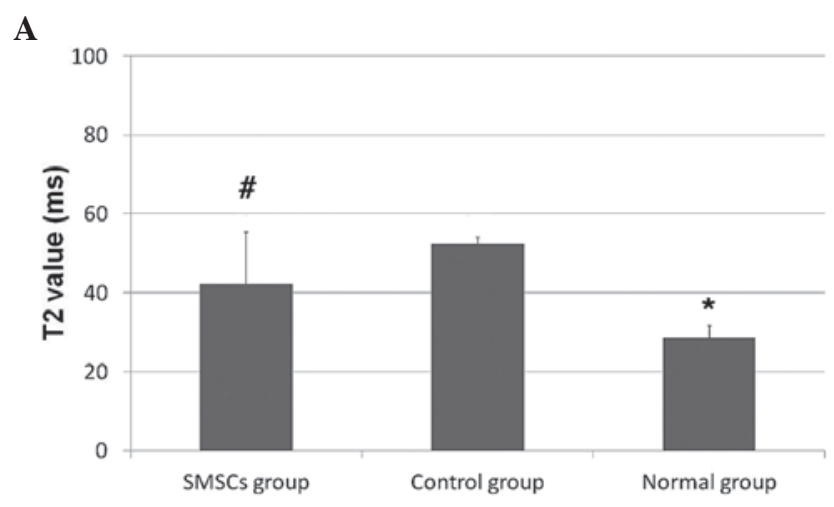

B

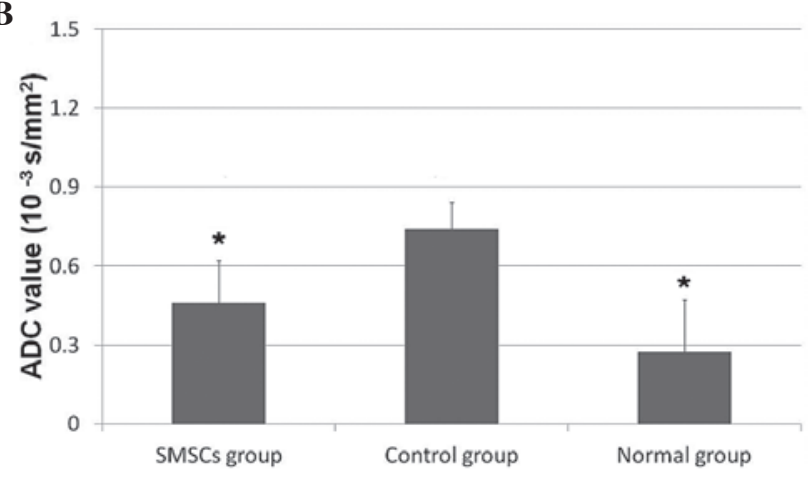

Figure 3. Comparison of $\mathrm{T} 2$ and $\mathrm{ADC}$ values. (A) T2 values of the norma group, OCD group and SMSC group. (B) Comparison of apparent ADC values among the normal group, OCD group and SMSC group. Data are presented as the mean \pm standard deviation. ${ }^{*} \mathrm{P}<0.05$ vs. the control group, ${ }^{\#} \mathrm{P}<0.05$ vs. the normal group. OCD, osteochondral defect. SMSCs, synovial membrane-derived mesenchymal stem cells; ADC, apparent diffusion coefficient.

showed areas of long $\mathrm{T}_{2}$ components at the cartilage lesion, particularly in the subchondral region. At 12 weeks following SMSC transplantation, the repair tissue of the SMSC group also showed a significantly higher $\mathrm{T}_{2}$ value, compared with the normal cartilage group $(42.26 \pm 13.09$, vs. $28.60 \pm 3.20 \mathrm{~ms}$; $\mathrm{P}=0.0283$; Fig.3A). The repair tissue in the SMSC group had a shorter $\mathrm{T}_{2}$, compared with that of the control group, although no significant difference was detected ( $P>0.05$; Fig. 3A). No significant variation in $T_{2}$ values were observed from the deep to superficial cartilage between the control and SMSC groups. All three groups exhibited areas of short $\mathrm{T}_{2}$ components in the superficial cartilage.

In the DWI imaging, the repair tissue in the SMSC group exhibited a significantly lower ADC value, compared with the control group $\left(0.46 \pm 0.16 \times 10^{-3}\right.$, vs. $0.74 \pm 0.10 \times 10^{-3} \mathrm{~s} / \mathrm{mm}^{2}$; $\mathrm{P}=0.016$; Fig.3B). No significant differences were observed between the ADC values of the SMSC group and the normal group $\left(0.46 \pm 0.16 \times 10^{-3}\right.$, vs. $0.27 \pm 0.20 \times 10^{-3} \mathrm{~s} / \mathrm{mm}^{2} ; \mathrm{P}=0.17$; Fig. 3B).DWI imaging of the normal group showed clear signal variation in the normal cartilage, with a signal transition between low and high ADC values observed between the deep and superficial cartilage (Fig. 4). However, cartilage repair tissue in the control group showed no signal variation in the ADC values, suggesting a lack of correct collagen arrangement. The repair tissue area of the SMSC group showed no transition between low and high ADC values between the deep and superficial articular layers of the repair site.

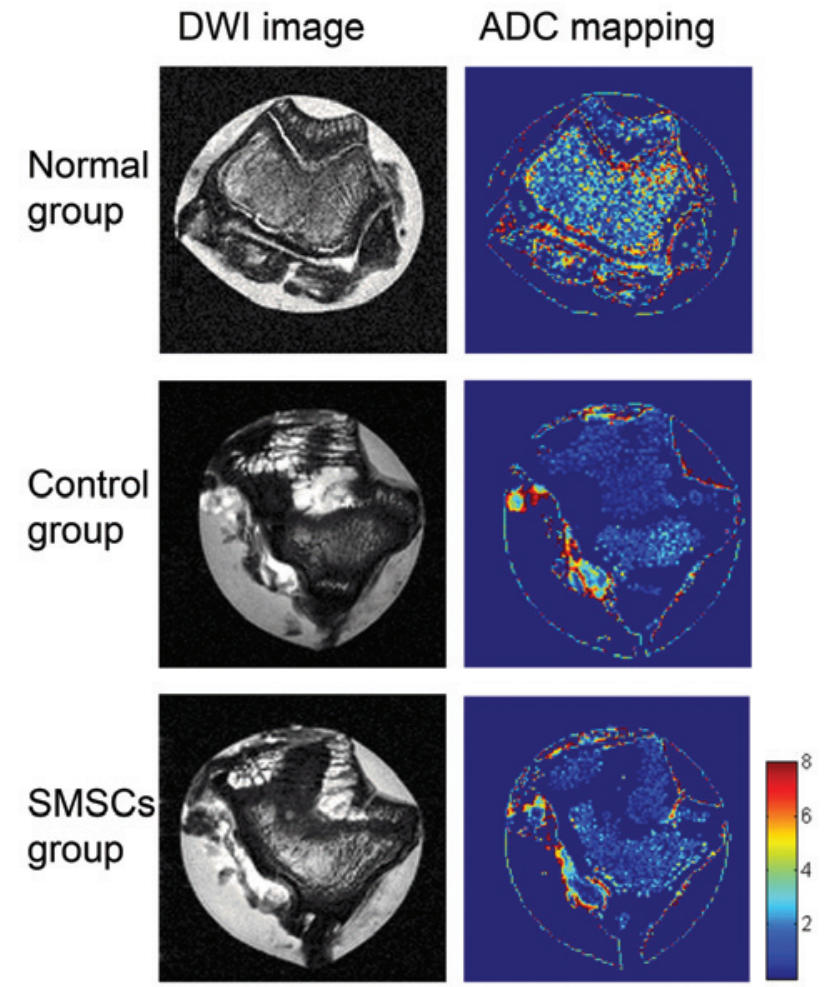

Figure 4. ADC map of cartilage repair tissue in the normal, OCD and SMSC group $\left({ }^{*} 10^{-4} \mathrm{~mm}^{2} / \mathrm{s}\right)$. Normal hyaline cartilage exhibited zonal variation, with a transition between low and high ADC values observed from the deep to superficial regions. Higher color values indicate areas of poor cartilage repair. OCD, osteochondral defect; SMSC, synovial membrane-derived mesenchymal stem cells. ADC, apparent diffusion coefficient.

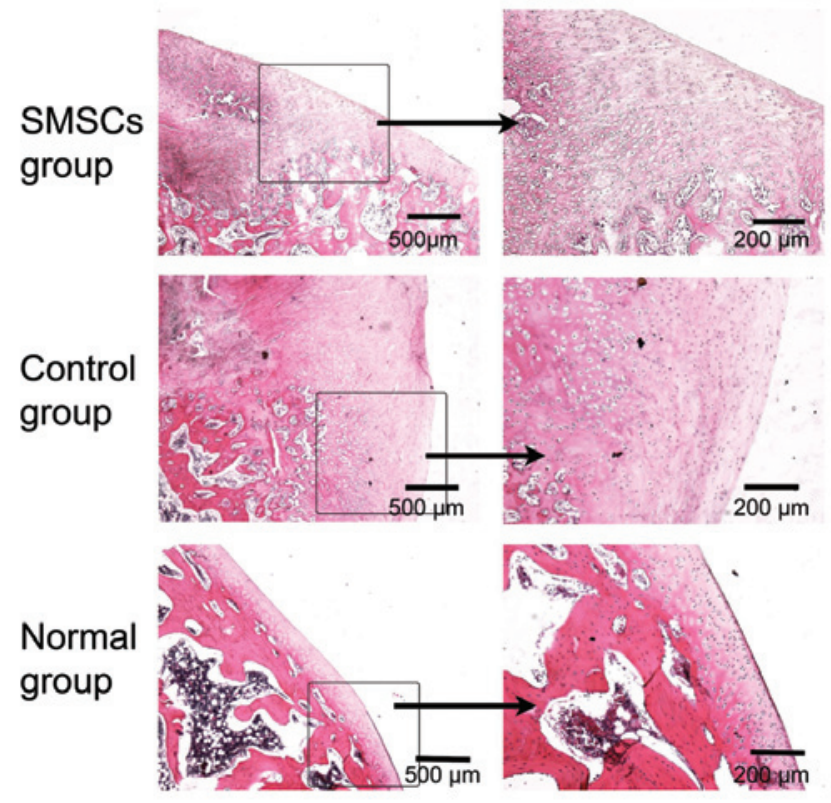

Figure 5. Histological analysis of repair tissue samples stained with hematoxylin and eosin 12 weeks following surgery to induce OCD. Staining indicates the cartilage defect in the tissue. OCD, osteochondral defect; SMSCs, synovial membrane-derived mesenchymal stem cells.

Histological results. As determined by the histological analysis, the cartilage tissues in the normal group were compact and exhibited a structural arrangement (Fig. 5). At 12 weeks 


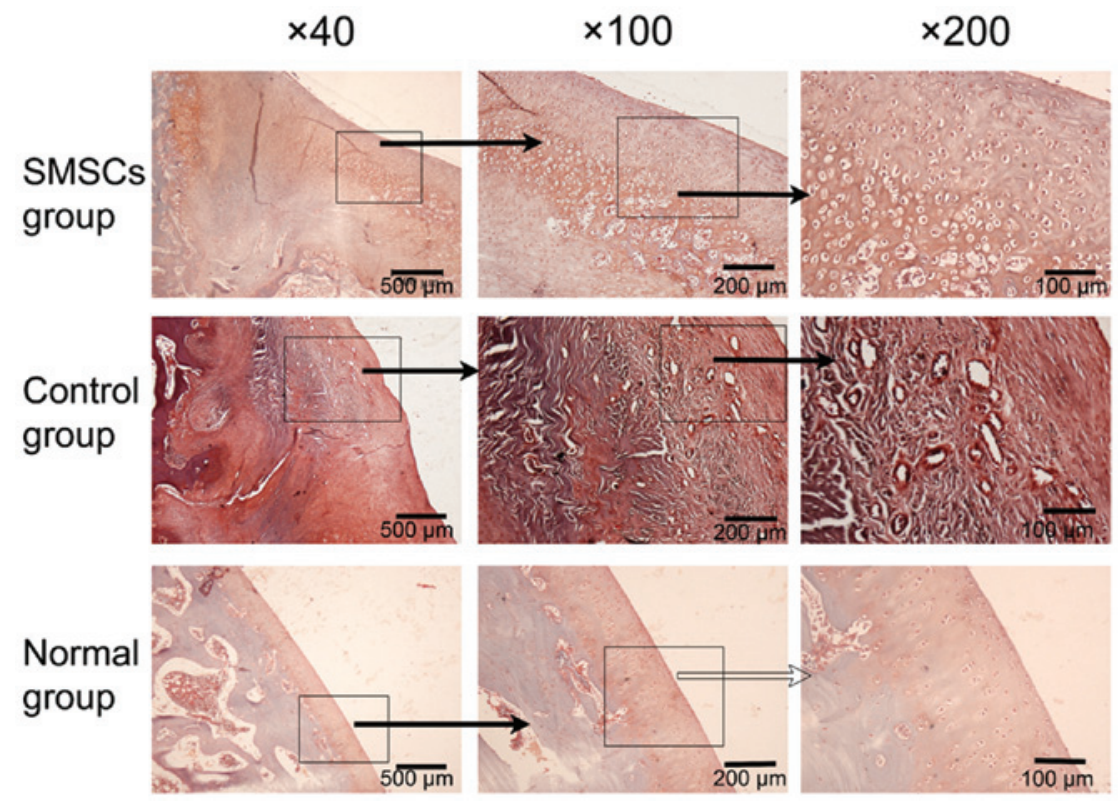

Figure 6. Histological analysis of repair tissue samples stained with Safranin-O 12 weeks following surgery to induce OCD. The images indicate the osteochondral repair using SMSCs displayed the appropriate tissue texture. OCD, osteochondroal defect; SMSCs, synovial membrane-derived mesenchymal stem cells.

A

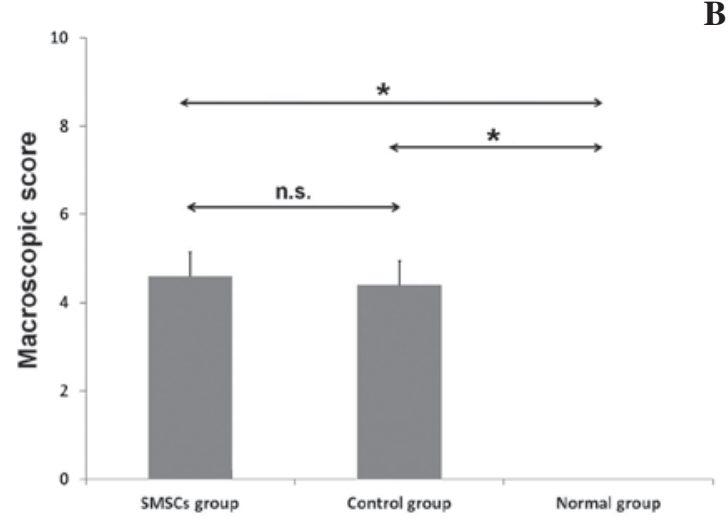

B

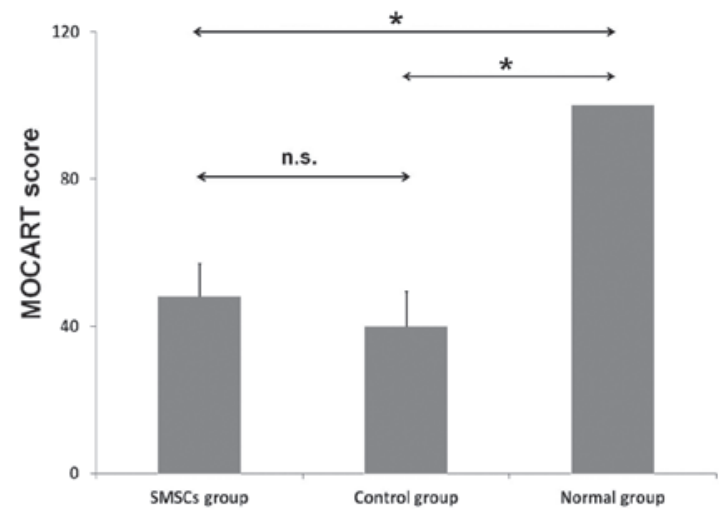

C

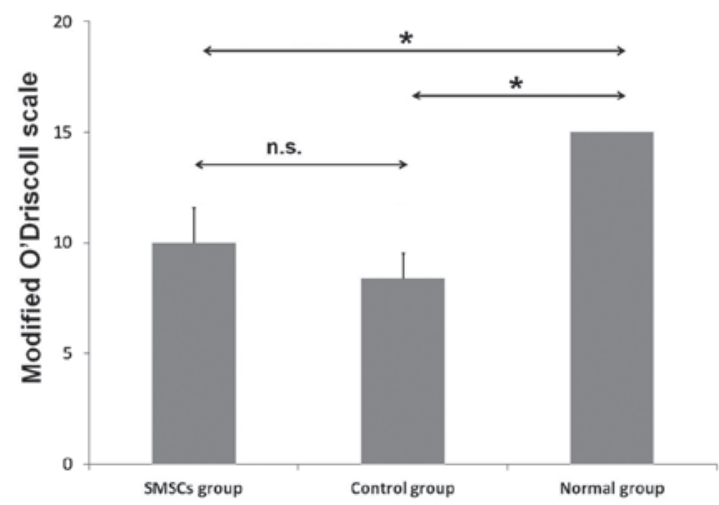

Figure 7. Assessment of tissue repair. (A) Macroscopic scores of cartilage repair 12 weeks post-surgery. (B) 2D MOCART scores of cartilage repair 12 weeks post-surgery. (C) Modified O'Driscoll scale of cartilage repair 12 weeks post-surgery. Data are presented as the mean \pm standard deviation. No significant differences were observed between the OCD and SMSC group in macroscopic score, 2D MOCART score or modified O'Driscoll scale. "P<0.05. OCD, osteochondral defect; SMSCs, synovial membrane-derived mesenchymal stem cells; MOCART, magnetic resonance observation of cartilage repair tissue; n.s., not significant.

following the SMSC transplantation procedure, the defects in the SMSC group and the control group had been filled with repair tissues. In the control group, fibrous repair tissue penetrated deeply into the subchondral bone, however, there was no evidence of hyaline-like cartilage or restored cartilage surface, and a severe fissure was observed at the cartilage 
interface. The defects were predominantly filled with granulation and fibrous tissue, which exhibited poor Safranin-O staining (Fig. 6). In the SMSC group, repair tissue covered the lesion site of the osteochondral samples. Fibrous tissue was also observed in the superficial layer of the SMSC group, and subchondral bone formation was present. Small, flat cells were observed in the surface layer, and large, round cells were located in the deep layer (Fig. 5). Safranin-O staining revealed repair tissues, which were stained red and regularly distributed in the SMSC group, which was indicated by the columnar alignment of the cells in the deep layer and the small tangentially organized cells in the superficial area (Fig. 6).

The macroscopic score and 2D MOCART score, and the modified O'Driscoll scale are shown in Fig. 7. No significant differences were observed between the control group and the SMSC group in the macroscopic score $(\mathrm{P}>0.05)$, the $2 \mathrm{D}$ MOCART score $(\mathrm{P}>0.05)$ or the modified O'Driscoll scale $(\mathrm{P}>0.05)$.

\section{Discussion}

In the present study, tissue repair following SMSC transplantation in a rabbit osteochondral defect model was analyzed using 9.4T high-field MRI. The DWI technique revealed unique SMSC healing outcomes, demonstrating regional variation in cartilage organization. The SMSC group exhibited increased cartilage healing, compared with the control group, resulting from the production of the regularly distributed organization of repair tissue at the repair site.

Previous investigations have demonstrated that MSC grafts improve the early healing response, increasing collagen type II-positive cross-sectional areas of the regenerated tissue and increasing aggrecan content $(6,22)$. It has also been demonstrated that intra-articular bone marrow-derived MSCs enhance cartilage repair quality, with increased aggrecan content and tissue firmness (23). In the present study, it was observed that SMSC implantation increased repair tissue quality, with columnar alignment of cells in the deep layer and small tangentially organized cells in the superficial layer.

In addition, MRI at 9.4T revealed that the signal intensity of the osteochondral defect site was higher than that of the adjacent cartilage. However, graft signal intensity was not directly associated with graft histological appearance (24). Therefore, $\mathrm{T}_{2}$ mapping and the DWI technique were subsequently used to differentiate the repair tissue. $\mathrm{T}_{2}$ mapping uses the water content of cartilage and its transverse relaxation time as biochemical markers for changes in cartilage collagen structure $(25,26)$. A significant trend of increasing $\mathrm{T}_{2}$ values, from the deep to the superficial layer, is found in hyaline cartilage, whereas fibrous tissue sites exhibit no significant change in $\mathrm{T}_{2}$ value with depth $(13,27)$. In the present study, the cartilage of the normal tissue specimens revealed a homogenous $\mathrm{T}_{2}$ pattern, with no significant regional variation between the deep and superficial cartilage. All three groups exhibited areas of short $\mathrm{T}_{2}$ in the superficial cartilage, possibly owing to the effect of neutral-buffered formalin on the cartilage.

DWI exploits the mobility of water protons in biological tissues, and thus can reveal the structure of biological tissue at a microscopic level $(16,18,28)$. Whereas $\mathrm{T}_{2}$ mapping is specific for collagen network and water-content, DWI is specific for collagen and proteoglycan content (29). Mean diffusivity, evidence by the ADC, is linearly correlated to progressive proteoglycan extraction in articular cartilage (30). The ADC value of normal cartilage increases from the bone-cartilage interface to the articular surface (12). In the present study, DWI imaging of the normal group showed marked regional variation, with a transition between low and high ADC values from the deep to superficial regions. It was hypothesized that the DWI reflects the biochemical constitution of cartilage as a combination of collagen content/orientation, hydration and glycosaminoglycan content (18). Furthermore, in the repair tissue area of the SMSC group, no transition between low and high ADC values were observed between the deep and superficial areas. These results suggested that SMSC implantation alone was not able to restore the native order of cartilage structure, however, it increased the columnar alignment of cells in deep layer and small tangentially organized cells in the superficial layer. Although the repair tissue area of the SMSC group had a significantly lower ADC value, compared with the control group, no significant difference in ADC values were observed between the SMSC and the normal group. Therefore, the ADC maps provided a more sensitive technique for the detection of improved repair tissue quality in the SMSC group.

The present study presented with a number of limitations. Firstly, the cartilage defect samples were examined ex vivo, therefore, the results cannot be generalized to in vivo conditions. Secondly, the present study evaluated only a single time point, with a small number of samples. Future in-depth investigations with a larger sample size is required to provide valuable information regarding the validity of these results. Finally, there was the lack of direct histological correlation for the $\mathrm{T}_{2}$ and ADC measurements.

In conclusion, the present study demonstrated that osteochondral repair using SMSCs facilitated the repair of appropriate tissue structure. In addition, clinical scoring, morphological MRI, $\mathrm{T}_{2}$ mapping and DWI at 9.4T high field provided additional information in the evaluation of cartilage repair tissue quality. The DWI technique may be applied to evaluate the treatment progress of osteochondral lesion.

\section{Acknowledgements}

This study was supported by the Nano Project of Shanghai Municipal Science and Technology Commission (grant nos. 1052nm03701 and 11nm0504900), the Project of Shanghai Municipal Science and Technology Commission (grant no. 11JC1401700) and the National Natural Science Foundation of China (grant nos. 81201068, 81472142, 81401812 and U1232212).

\section{References}

1. Harris JD, Siston RA, Pan X and Flanigan DC: Autologous chondrocyte implantation: A systematic review. J Bone Joint Surg Am 92: 2220-2233, 2010.

2. Bekkers JE, Tsuchida AI, van Rijen MH, Vonk LA, Dhert WJ, Creemers LB and Saris DB: Single-stage cell-based cartilage regeneration using a combination of chondrons and mesenchymal stromal cells: Comparison with microfracture. Am J Sports Med 41: 2158-2166, 2013.

3. Roelofs AJ, Rocke JP and De Bari C: Cell-based approaches to joint surface repair: A research perspective. Osteoarthritis Cartilage 21: 892-900, 2013. 
4. Lee KB, Hui JH, Song IC, Ardany L and Lee EH: Injectable mesenchymal stem cell therapy for large cartilage defects-a porcine model. Stem Cells 25: 2964-2971, 2007.

5. Sampat SR, O'Connell GD, Fong JV, Alegre-Aguarón E, Ateshian GA and Hung CT: Growth factor priming of synovium-derived stem cells for cartilage tissue engineering. Tissue Eng Part A 17: 2259-2265, 2011.

6. Jung M, Kaszap B, Redöhl A, Steck E, Breusch S, Richter W and Gotterbarm T: Enhanced early tissue regeneration after matrix-assisted autologous mesenchymal stem cell transplantation in full thickness chondral defects in a minipig model. Cell Transplant 18: 923-932, 2009.

7. Koga H, Muneta T, Nagase T, Nimura A, Ju YJ, Mochizuki T and Sekiya I: Comparison of mesenchymal tissues-derived stem cells for in vivo chondrogenesis: Suitable conditions for cell therapy of cartilage defects in rabbit. Cell Tissue Res 333: 207-215, 2008

8. Goebel L, Orth P, Müller A, Zurakowski D, Bücker A, Cucchiarini M, Pape D and Madry H: Experimental scoring systems for macroscopic articular cartilage repair correlate with the MOCART score assessed by a high-field MRI at 9.4 T-comparative evaluation of five macroscopic scoring systems in a large animal cartilage defect model. Osteoarthritis Cartilage 20: 1046-1055, 2012.

9. Rautiainen J, Lehto LJ, Tiitu V, Kiekara O, Pulkkinen H, Brünott $A$, van Weeren $R$, Brommer $H$, Brama PA, Ellermann J, et al: Osteochondral repair: Evaluation with sweep imaging with fourier transform in an equine model. Radiology 269: 113-121, 2013.

10. Madelin G,Babb J,XiaD,Chang G,Krasnokutsky S, Abramson SB, Jerschow A and Regatte RR: Articular cartilage: Evaluation with fluid-suppressed 7.0-T sodium MR imaging in subjects with and subjects without osteoarthritis. Radiology 268: 481-491, 2013.

11. Glaser C: New techniques for cartilage imaging: T2 relaxation time and diffusion-weighted MR imaging. Radiol Clin North Am 43: 641-653; vii, 2005 .

12. Raya JG, Horng A, Dietrich O, Krasnokutsky S, Beltran LS, Storey P, Reiser MF, Recht MP, Sodickson DK and Glaser C: Articular cartilage: In vivo diffusion-tensor imaging. Radiology 262: 550-559, 2012.

13. Welsch GH, Mamisch TC, Domayer SE, Dorotka R, Kutscha-Lissberg F, Marlovits S, White LM and Trattnig S: Cartilage T2 assessment at 3-T MR imaging: In vivo differentiation of normal hyaline cartilage from reparative tissue after two cartilage repair procedures-initial experience. Radiology 247: 154-161, 2008

14. Eshed I, Trattnig S, Sharon M, Arbel R, Nierenberg G, Konen E and Yayon A: Assessment of cartilage repair after chondrocyte transplantation with a fibrin-hyaluronan matrix-correlation of morphological MRI, biochemical T2 mapping and clinical outcome. Eur J Radiol 81: 1216-1223, 2012.

15. DomayerSE,Apprich S, StelzenederD, Hirschfeld C, Sokolowski M, Kronnerwetter C, Chiari C, Windhager R and Trattnig S: Cartilage repair of the ankle: First results of T2 mapping at 7.0 T after microfracture and matrix associated autologous cartilage transplantation. Osteoarthritis Cartilage 20: 829-836, 2012.

16. Friedrich KM, Mamisch TC, Plank C, Langs G, Marlovits S Salomonowitz E, Trattnig S and Welsch G: Diffusion-weighted imaging for the follow-up of patients after matrix-associated autologous chondrocyte transplantation. Eur J Radiol 73 : 622-628, 2010

17. Apprich S, Trattnig S, Welsch GH, Noebauer-Huhmann IM, Sokolowski M, Hirschfeld C, Stelzeneder D and Domayer S: Assessment of articular cartilage repair tissue after matrix-associated autologouschondrocyte transplantation or the microfracture technique in the ankle joint using diffusion-weighted imaging at 3 Tesla. Osteoarthritis Cartilage 20: 703-711, 2010.
18. Welsch GH, Trattnig S, Domayer S, Marlovits S, White LM and Mamisch TC: Multimodal approach in the use of clinical scoring, morphological MRI and biochemical T2-mapping and diffusion-weighted imaging in their ability to assess differences between cartilage repair tissue after microfracture therapy and matrix-associated autologous chondrocyte transplantation: A pilot study. Osteoarthritis Cartilage 17: 1219-1227, 2009.

19. De Bari C, Dell'Accio F, Tylzanowski P and Luyten FP: Multipotent mesenchymal stem cells from adult human synovial membrane. Arthritis Rheum 44: 1928-1942, 2001.

20. Marlovits S, Singer P, Zeller P, Mandl I, Haller J and Trattnig S: Magnetic resonance observation of cartilage repair tissue (MOCART) for the evaluation of autologous chondrocyte transplantation: Determination of interobserver variability and correlation to clinical outcome after 2 years. Eur J Radiol 57: 16-23, 2006

21. Zalewski T, Lubiatowski P, Jaroszewski J, Szcześniak E, Kuśmia S, Kruczyński J and Jurga S: Scaffold-aided repair of articular cartilage studied by MRI. MAGMA 21: 177-185, 2008.

22. Wilke MM, Nydam DV and Nixon AJ: Enhanced early chondrogenesis in articular defects following arthroscopic mesenchymal stem cell implantation in an equine model. J Orthop Res 25: 913-925, 2007.

23. McIlwraith CW, Frisbie DD, Rodkey WG, Kisiday JD, Werpy NM, Kawcak CE and Steadman JR: Evaluation of intra-articular mesenchymal stem cells to augment healing of microfractured chondral defects. Arthroscopy 27: 1552-1561, 2011.

24. Tins BJ, McCall IW, Takahashi T, Cassar-Pullicino V, Roberts S, Ashton B and Richardson J: Autologous chondrocyte implantation in knee joint: MR imaging and histologic features at 1-year follow-up. Radiology 234: 501-508, 2005.

25. Domayer SE, Kutscha-Lissberg F, Welsch G, Dorotka R, Nehrer S, Gäbler C, Mamisch TC and Trattnig S: T2 mapping in the knee after microfracture at 3.0 T: Correlation of global T2 values and clinical outcome-preliminary results. Osteoarthritis Cartilage 16: 903-908, 2008.

26. Kijowski R, Blankenbaker DG, Munoz Del Rio A, Baer GS and Graf BK: Evaluation of the articular cartilage of the knee joint: Value of adding a T2 mapping sequence to a routine MR imaging protocol. Radiology 267: 503-513, 2013.

27. White LM, Sussman MS, Hurtig M, Probyn L, Tomlinson G and Kandel R: Cartilage T2 assessment: Differentiation of normal hyaline cartilage and reparative tissue after arthroscopic cartilage repair in equine subjects. Radiology 241: 407-414, 2006.

28. Apprich S, Trattnig S, Welsch GH, Noebauer-Huhmann IM, Sokolowski M, Hirschfeld C, Stelzeneder D and Domayer S: Assessment of articular cartilage repair tissue after matrix-associated autologous chondrocyte transplantation or the microfracture technique in the ankle joint using diffusion-weighted imaging at 3 Tesla. Osteoarthritis Cartilage 20: 703-711, 2012.

29. Roemer FW, Crema MD, Trattnig S and Guermazi A: Advances in imaging of osteoarthritis and cartilage. Radiology 260: 332-354, 2011.

30. Raya JG, Melkus G, Adam-Neumair S, Dietrich O, Mützel E, Kahr B, Reiser MF, Jakob PM, Putz R and Glaser C: Change of diffusion tensor imaging parameters in articular cartilage with progressive proteoglycan extraction. Invest Radiol 46: 401-409, 2011 